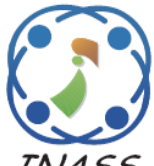

\title{
An Efficient Routing Protocol Adopting Enhanced Cluster Formation Technique Accompanied by Fuzzy Logic for Maximizing Lifetime of WSN
}

\author{
Nirmala Hiremani ${ }^{1 *}$ \\ Tiptur Gangaraju Basavaraju ${ }^{2}$ \\ ${ }^{I}$ Visvesvaraya Technological University, Mysore, India \\ ${ }^{2}$ Government SKSJ Technological Institute, Bangalore, India \\ *Corresponding author's Email: nirmalahiremani0375@gmail.com
}

\begin{abstract}
Wireless sensor networks is a system of multiple small sensors limited energy sources, used to sense any given sensing region of interest. In WSN, sensors might be required to work for a longer duration of time from the time of its deployment. Hence to optimize the utilization of energy at each node, we propose an algorithm, which can eventually enhance the lifetime and hence the reliability of the sensor nodes and the overall network. Here PSO based efficient clustering is performed and the appropriate selection of $\mathrm{CH}$ and SCH is done. The proposed approach outperforms the tradition F-LEAH algorithm. The result obtained through simulation shows that the proposed algorithm performs better than F-LEACH approach in terms of stability, reduced energy consumption at each node, less delay and drop at the BS and enhanced the lifetime of the network.
\end{abstract}

Keywords: Particle swarm Optimization; Fitness; GSA; Routing, Fuzzy logic; Clustering.

\section{Introduction}

Wireless technologies have revolutionized the world of communications. The world of communication has been raised by the Wireless technologies. In earlier days, the wireless technology was referred to the radio receivers or transceivers. But nowadays, the technologies such as the cellular networks and wireless broadband Internet is described in the wireless technology. The wireless medium is referred to the mobile communication which has limited spectrum along with a few other constraints. The indiscriminate and quick incorporation of a large number of nodes are made by the wireless ad hoc networking. The broad range of applications of wireless ad hoc network is tactical communications, disaster relief operations, health care and temporary networking. In the region which does not have a dense population, the temporary networking is utilized.

The other name for Wireless Sensor Networks (WSN) is Wireless Sensor and Actuator Networks
(WSAN) [1]. WSN consists of self-directed sensor nodes which are spatially disseminated to examine the physical and environmental circumstances. The environmental circumstances include temperature, sound, pressure, etc. By using the network, the WSN is used to transfer the data to the main location. The military applications such as battlefield surveillance prompt the progress of wireless sensor networks.

The broad range of applications in military and national security, environmental screening, and many other fields has made the sensor nodes in the network as available in the low-power wireless integrated micro sensor technologies and latest developments [2]. A flexible scheme is offered by the sensor networks which are different from traditional sensors with simple deployment and numerous functionalities. The position of the nodes in the classical sensors and the topology of the network should be fixed and designed with care [3]. The research in WSN focused on energy consumption and enhanced the lifetime of the network in the given location. In the establishment of a 
network in the linked hierarchy, the clustering mechanism forms an efficient topology control methodology which stabilizes the traffic load and improves the overall performance and also increases the lifetime of WSNs. The major goal of this research is to establish an energy efficient routing technique which supplies reliable communication by considering the above-declared routing challenges and enhance the lifetime of WSNs.

Main contribution of our work includes:

- Efficient clustering in the given sensing are without any residual node left without being a member node.

- Efficient $\mathrm{CH}$ and in turn selecting a Super Cluster head from among the selected $\mathrm{CH}$ such that only $\mathrm{SCH}$ is eligible to directly communicate with the base station.

- Using GSA approach to efficiently select the route from a cluster to its $\mathrm{CH}$, which then routes the data to the BS through the selected $\mathrm{SCH}$.

The rest of the paper is organized as follows: Section 2: presents an idea of the related works in the field of WSN. Section 3 provides detailed proposed approach. Results and discussions are provided in section 4 . Section 5 included the conclusion and future work.

\section{Literature Review}

Farouk et al [4] have proposed a protocol for heterogeneous WSNs. The proposed protocol is named as a Stable and Energy Efficient Clustering (SEEC). An extension to the multi-level SEEC is also described. It depends on the network structure which is fragmented into clusters. In this cluster, some normal nodes are incorporated randomly. A powerful advanced node is presented in each of the clusters. In order to envelop the distant sensing areas, more number of powerful super nodes is assigned in the multi-level architectures. Each node in the network has the role of sensing, aggregation or transmission to the base station. The minimum energy consumption of the network is achieved by the optimum number of powerful nodes at each level of heterogeneity. The proposed protocol is essential for many applications as it is a heterogeneous awareness protocol for prolonging the stability period. The existing homogeneous and heterogeneous protocols are compared with the performance of the proposed protocol. The simulation results show that longer stability period, more energy efficiency and higher average throughput are provided by the proposed protocols. When the number of rounds has been increased for each set of node, residual energy of nodes has been decreased. Chand et al [5] have proposed HEED for the heterogeneous network. It depends on the type of nodes. Here, one-level heterogeneity is referred as hetHEED-1, two-level heterogeneity is referred as hetHEED-2 and threelevel heterogeneity is referred as hetHEED-3. Consequently, distance is considered as the additional parameter. While determining the cluster heads, the fuzzy logic is applied. By this, hetHEED-1 is named as HEEDFL, hetHEED-2 is named as hetHEED-FL-2 and hetHEED-3 is named as hetHEED-FL-3. The simulation results show that the decrease of the rate of energy dissipation, an increase of heterogeneity level in the network and the nodes remain alive for a longer period. In addition to this, the increased heterogeneity level increases the network lifetime and helps in sending more packets to the base station. Often, the increase in the network energy increases the network lifetime. In the case of using fuzzy logic, the network lifetime increases by $114.85 \%$ when compared to the original HEED, it also does not increase the network energy. Henceforth, $387.94 \%$ of increased lifetime is provided by the hetHEED-FL-3 when the network energy increases by $19 \%$. Consequently, it sends a maximum number of packets to the base station and has a minimum rate of energy abundance. The main drawback of this proposed method is utilization of overhead or addition of extra bits has been increased. Zeng and Dong [6] have proposed an algorithm namely Improved Harmony Search Based Energy Efficient Routing (IHSBEER) algorithm for WSNs. This algorithm is based on the meta-heuristic algorithm namely, Harmony search (HS). These key improvements are as follows. First is, encoding the harmony memory depending on the characteristics of the routing in WSNs. The second one is the improved New Harmony. In early generations, a dynamic adaptation for the parameter HMCR is introduced to avoid the prematurity. Additionally, it strengthens its local search ability in late generations. In order to make the less parameter in the proposed routing algorithm, the adjustment process of HS algorithm is discarded. The third one is, to enhance the local search ability, to improve the accuracy of the routing algorithm and to improve the convergence speed, an effective local search strategy is proposed. Consequently, the energy consumption and the length of the path are considered by the developed objective 
function. It also includes the detailed descriptions and test results of the performance of the proposed approach. The experimental results show the advantages of the proposed routing algorithm for WSNs. But throughput of this proposed algorithm was not efficient.

\section{Proposed Solution}

\subsection{System Design}

In our approach, a wireless sensor network in an area of interest is considered (with multiple applications from sensed surrounding). Base Station (BS) is employed in the sensing area to collect the information from the wireless sensors (particles). During an event if interest (such as flooding, earthquake...etc) the sensor nodes deployed unevenly and with mobility must send the data efficiently to its BS (in the sensing region) with high efficiency and reliability.

In the proposed approach an efficient clustering and routing is established. Between each sensor and its Base Station. Let us consider:

- Base station is not mobile and has abundant

Bandwidth and energy source

- Sensor nodes are mobile and have limited energy source

\subsection{Cluster Member Selection using Particle Swarm Optimization (PSO)}

In the proposed protocol Particle Swarm Optimization (PSO) is employed for efficient clustering of nodes with least redundant (un-clustered) node in the network. Particle Swarm Optimization (PSO), devised by Eberhart and Kennedy in 1995, is a random optimization technique based on population. PSO is a computational technique that optimizes a problem (situation) using a series of iterations to enhance the possible solution for a given quality measure A solution to the problem of complex nonlinear optimization has been proposed using PSO by means of imitating the bird flocks behavior.

PSO simulates the behavior of flocking birds, where a group of birds randomly search food in a given area. Consider there is only one piece of food in the area of search and no birds know the where the food is. In each iteration, velocity of each particle is updated using the current velocity and the previous local_best and global_best position. Based on them the new_velocity and new_position values are updated. The first best maximum value is known as the pBest. Then the second best value is evaluated keeping the best value so far obtained in the whole swarm population as the gBest (global best).

With the help of PSO algorithm during cluster formation, some of the nodes which were left out without being a member of any cluster which results in residual node formation, clustering is carried out until all the nodes become a member of any of the present clusters. Hence the rate of individual node formation which reduces the lifetime of the network is eliminated. Initially, when all the nodes are deployed in the network, the base station (sink) broadcasts an INITIATE_MSG followed by an INFO_COLLECT message to all the sensor nodes in the network. The sensor nodes after receiving the INITIATE_MSG and the INFO_COLLECT message from the base station (sink), starts to communicate with every other sensor node in the give sensing region, by broadcasting anHELLO_message, in their sensing region.

After the broadcast of the HELLO_message, every sensor node sends an INFO_COLLECT reply message to the base station_HELLO_message contains information about the nodes id and its residual energy, bandwidth and connectivity. INFO_COLLECT reply message sent from the $n$th sensors to the sink node (base station) contains the following information:

- Position $\left(u_{n}, w n\right)$ of the sensor node.

- Velocity $\left(v_{1 n}, v_{2 n}\right)$ of the sensor nodes. (where $v_{I n}$ is the current velocity of node $n, v_{2}$ is the average velocity of node $n$ )

- $\quad$ Energy $\left(E_{n}\right)$ of the $n$th sensor node.

Hence for each sensor node the value of position, velocity and energy are maintained and updated at the base station (sink node).

In this proposed algorithm, each WSN sensor node is considered as the particle. Here the base station (sink node) makes the sensor nodes to perform cluster formation, which is carried out using Particle Swarm Optimization (PSO).

Using PSO, the fitness of each particle is calculated to choose the cluster particles (cluster member selection). Fitness value in our proposed approach depends on:

- The energy of the sensor node (particle) $E_{m}$.

- Connectivity $\left(C_{m n}\right)$

- Distance of sensor node/particle $(n)$ within the radio range ' $a$ ' from node $m$ 
- Energy of the sensor node/particle $(n)$ within the radio range ' $a$ ' from node $m$

Based on the fitness value clustering is carried out considering all the sensor node and hence eliminating the presence of residual nodes in the network. The nodes with maximum number of connectivity and residual energy are considered as the cluster particle (member).

$$
F=k_{1} \times\left(D_{n m} \times U\right)+k_{2} \times\left(\frac{E_{A v g_{c m n}}}{E_{m}}\right)+{ }_{k_{3}} \times U
$$

Where, $U=\frac{1}{C_{m n}}$

$$
D_{n m}=\sqrt{\left(u_{m}-u_{n}\right)^{2}+\left(w_{m}-w_{n}\right)^{2}} \text { i.e. the distance }
$$

between node $m$ and $n$th node

$n=\{1,2,3 \ldots . l\}$ be the number of nodes (particles) reachable by the given node $p$ in the given sample space $0<k_{1}<1,0<k_{2}<1$ and $k_{3}=1-k_{1}-k_{2}$,

Fitness value of each node is calculated during every iteration and the maximum fitness value obtained is taken as the local_best. The maximum value among all the fitness values obtained is taken as global_best. Position and velocity updation is carried out in PSO for every particle as follows:

$$
V_{p}=W_{v_{p}}+W_{1}\left(P_{\text {pre }}-P_{\text {cur }}\right)+W_{2}\left(P_{\text {pre }} \times P_{\text {cur }}\right)
$$

$$
P_{-} \text {update }_{p}=\text { Previousposition of node } p+V_{-} \text {update }_{p}
$$

Where,

$W_{v p}=$ weight of nodes velocity, $W_{l}$ and $W_{2}=$ weight of nodes location, Ppre represents previous position of node $\mathrm{P}$ and $P_{c u r}$ represents current position of node $\mathrm{P}$

As shown in Fig.1, $(X, Y)$ be the assumed workspace (sensing region) and ' $a$ ' be the coverage of each sensor nodes. The total number of cluster formed in the considered sensing area can be calculated as:

$$
N=\frac{\text { total network area }}{\text { individualcluster area }}=\frac{X \times Y}{x \times y}
$$

Where,

$(x, y)$ be the coordinates of the cluster in the sensing region, $(X, Y)$ be the coordinates of the sensing region

From the Fig.1, using Pythagoras theorem the value of a can be written as:

$$
a=\sqrt{\frac{x^{2}}{4}+\frac{y^{2}}{4}}
$$

Let us assume, $x=y=u$ and $X=Y$. Equation (1) and (2) becomes:

$$
\begin{aligned}
a & =\frac{u}{\sqrt{2}} \\
N & =\frac{(X \times Y)}{u^{2}}=\frac{(X \times Y)}{2 a^{2}}
\end{aligned}
$$

The upper bound of the number of clusters formed in a network can be:

$$
N=\left\{\left(\frac{X \times Y}{x \times y}\right)+\left(\frac{X}{x}\right)+\left(\frac{Y}{y}\right)\right\}
$$

Substituting, $x=y=u$ and $X=Y$ in Eqn. (5)

$$
N=N=\frac{\left.\left(X^{2}\right)+(2 \sqrt{2} \times x \times a)\right)}{2 a^{2}}
$$

\subsection{Cluster Head Selection}

In this section we propose am modified energy efficient $\mathrm{CH}$ selection technique based on parameters like Residual energy, distance to the neighbor, density, maximum distance and angle. The core aim of this technique is to minimize the heavy data traffic and high energy consumption in the network. The development technique fixes each $\mathrm{CH}$ near to the sink and sensing event while the remaining set of the $\mathrm{CHs}$ are appointed in the middle of each cluster to achieve the highest level of energy efficiency in a deployed dense network.

- Residual energy $\left(\boldsymbol{R} \boldsymbol{E}_{n}\right)$ : A cluster head should have enough energy to perform packet transmission, Witness data flow and process the data.

$\mathrm{CH}_{R E}>$ Threshold.

- Maximum distance: is the maximum distance between node $m$ and node $n$ i.e. $D_{n \max }$

- Angle: Angle of the sensor node $m$ to its neighboring node $n$

- Density $(\rho)$ : Density $(\rho)$ represents the number of nodes (sensors/particles) in a given region. $\rho=1$ for thick region, $\rho=2$ for thin region

- Distance to the Neighbor $\left(D_{n m}\right)$ : A CH must have smallest transmission distance to all its member nodes in the given densely deployed cluster. Hence, the $\mathrm{CH}$ must efficiently cover the given sensing region with least amount of energy consumption.

Active nodes in a region ' $a$ ' around a node to select itself as the $\mathrm{CH}$ can be calculated using: 


$$
\text { Activenodes }=\frac{R E_{n}}{\left(\sum_{i=1}^{n} \frac{D i m}{\operatorname{Dim}(\max )}\right)^{2}+\left(\rho-\left(\frac{D i}{100}\right)\right)^{2}}
$$

Algorithm 1: Cluster head selection

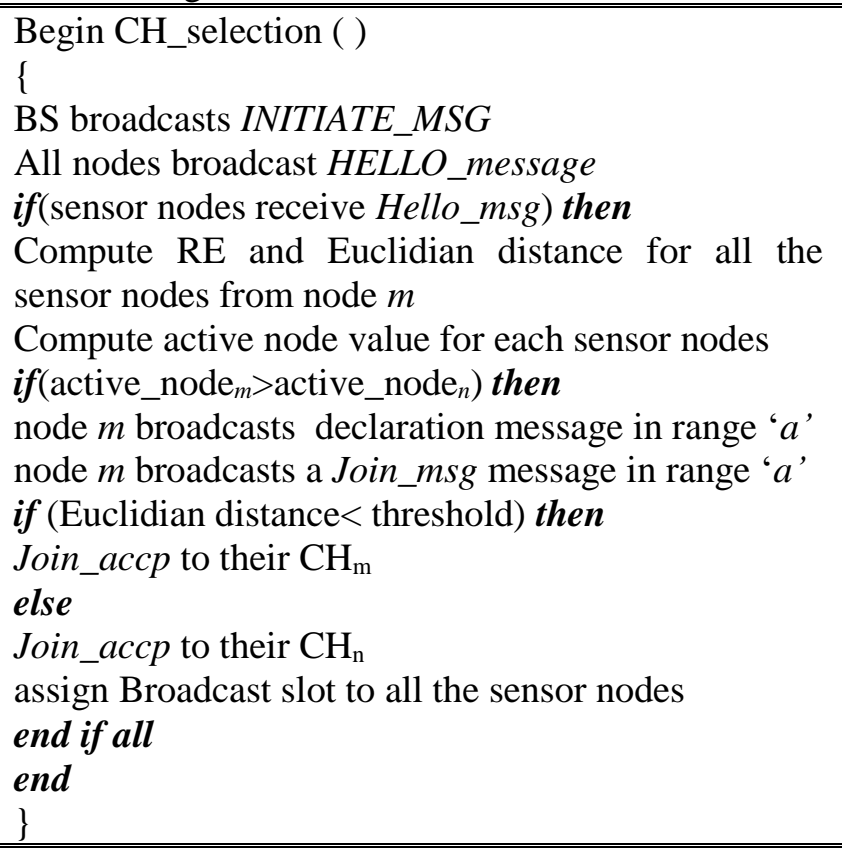

\subsection{SCH Selection}

Sensor nodes send the sends data in a given environment after detecting an event (interference). $\mathrm{CH}$ collects the information and aggregates the data before sending it to the bases station. As the $\mathrm{CH}$ consumers more energy in sensing and broadcasting the message the lifetime of $\mathrm{SH}$ decreases and hence leads to a reduction in the usefulness of the cluster (which contains the $\mathrm{CH}$ with low residual energy). Hence to increase the lifetime of the cluster and to increase the lifetime of the cluster head, we propose to elect a super cluster head $(\mathrm{SCH})$ in the considered sensing region (area considered) as shown in fig. 2. These Super Cluster Head are selected among the selected $\mathrm{CH}$ and can directly send the data directly to the BS using fuzzy interference engine (Mamdani's rule) by choosing fuzzy descriptors such as remaining battery power, the mobility of $\mathrm{BS}$, the centrality of the cluster, and energy dissipation ratio.
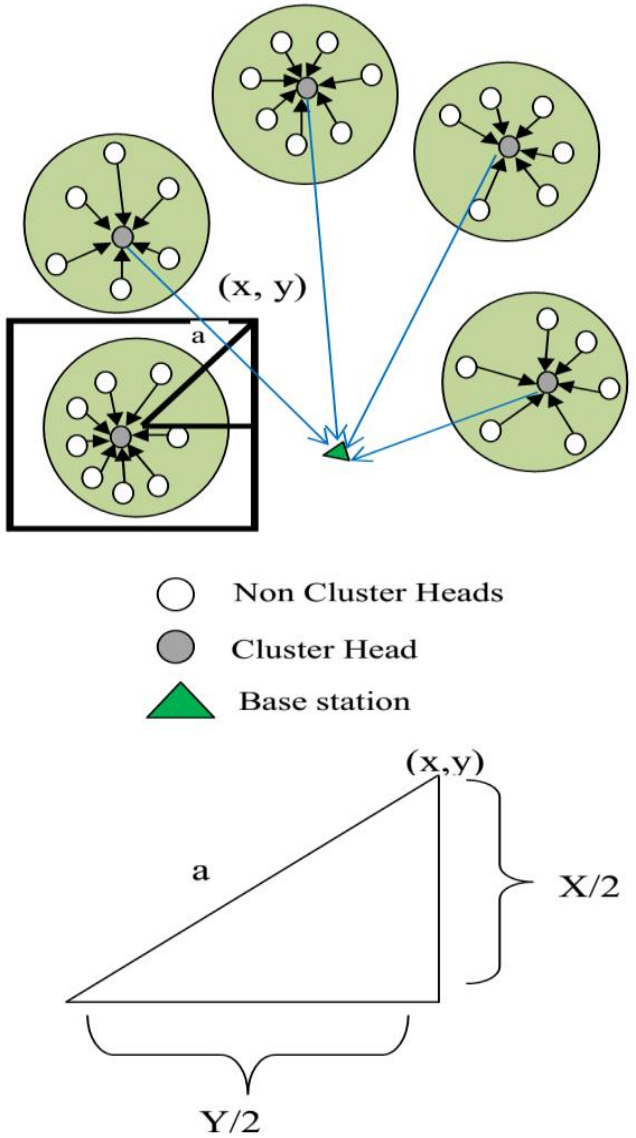

Figure.1 Clustering and $\mathrm{CH}$ selection using PSO

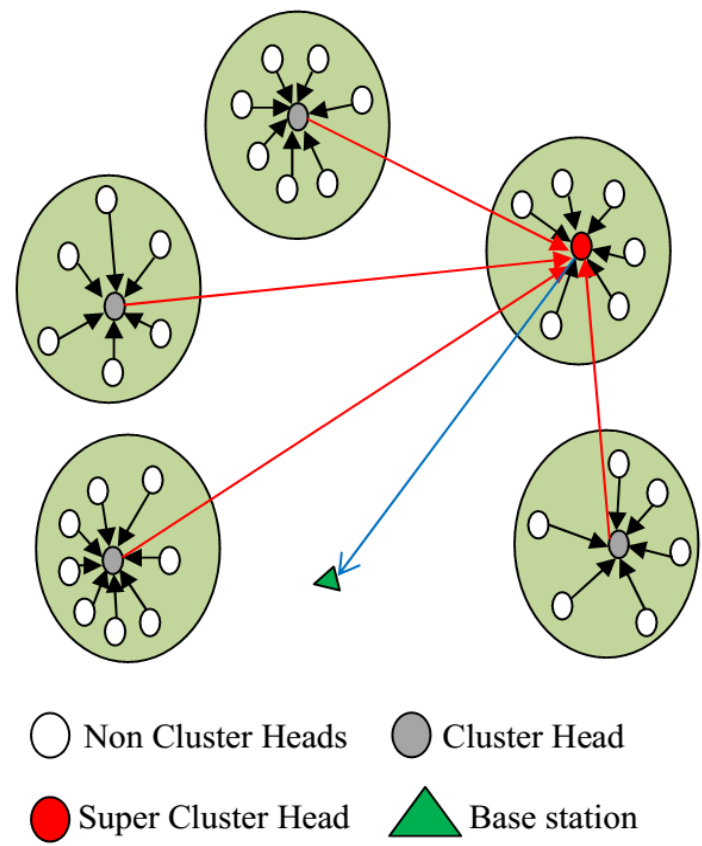

Figure.2 SCH selections using Fuzzy logic 
As only SCH can directly send the data to the BS, bandwidth utilization is effective. Here instead of utilization of multiple $\mathrm{CHs}$, only a single $\mathrm{SCH}$ is utilized, this can hence enhance the energy efficiency and reduce the energy consumption, to account to increase in the overall lifetime of the network.

\subsubsection{Fuzzy based SCH Selection}

In this proposed approach, Mamdani's fuzzy approach is used as shown in Fig.3. The fuzzy model consists of four processes:

1. Fuzzification: In fuzzification, inputs are given with crisp value and changed into fuzzy sets.

2. Rule Evaluation: The fuzzified inputs are taken and applied to the antecedents of the Fuzzy rules. It is then applied to the consequent membership function.

3. Aggregation of the rule outputs: This involves the merging of the output of all rules.

4. Defuzzification: Defuzzifier transforms the fuzzy set into a crisp value.

The linguistic variables for the fuzzy set are set less, medium and high. Both trapezoidal and triangular membership functions are employed for less, high and medium variables respectively. The fuzzy rules are driven by the formula:

$$
\begin{aligned}
& S C H_{\text {eligibility }}=\operatorname{Re} \text { maining } \\
& + \text { BatteryPower } \\
& + \text { mobility }+ \text { centrality }+ \text { Energydissipationratio }
\end{aligned}
$$

Re maining ${ }_{\text {BatteryPower }}=($ BatteryPoner -1$)(12)$

In Eq. (11) the value of $S C H_{\text {eligibility }}$ is calculated using the values of battery power (Pr), mobility (Mo), centrality (Cen) and energy dissipation ratio (EDR). Since during each information exchange, a nodes consumes energy, remaining battery power of a node decreases. Hence Eq. (12) is used to calculate the remaining battery power at a node. Whereas both mobility and centrality are considered to be an additive factor, since the distance of $\mathrm{SCH}$ from the BS increases or decreases as the BS moves.

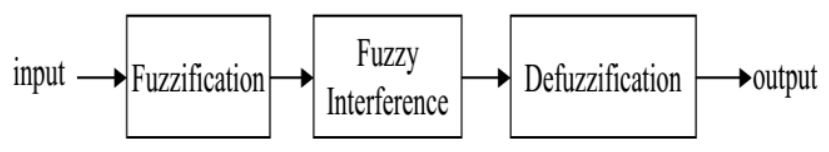

Figure.3 Fuzzy Logic system
Table 1. Fuzzy Rule for selection of SCH from multiple $\mathrm{CHs}$

\begin{tabular}{|c|c|c|c|c|}
\hline Pr & Mo & Cen & EDR & SCH \\
\hline$(1)$ & $(2)$ & $(2)$ & $(1)$ & $(5)$ \\
\hline$(3)$ & $(1)$ & $(2)$ & $(0)$ & $(4)$ \\
\hline$(3)$ & $(2)$ & $(2)$ & $(1)$ & $(5)$ \\
\hline
\end{tabular}

Centrality value depicts the connectivity of the $\mathrm{CH}$ with respect to other $\mathrm{VHs}$ in the given sensing region. $S C H_{\text {eligibility }}$ value of a node depicts the chances of a node being an $\mathrm{SCH}$ in the given network. The output of $S C H_{\text {eligibility }}$ is composed of seven membership functions: Very weak (-1), Weak (0), Lower medium (1), Medium (2), Higher medium (3), Strong (4), Very strong (5).

A $\mathrm{CH}$ has a high chance of being an $\mathrm{SCH}$ only if the conditions in Table 1, from among multiple conditions are satisfied. Hence, using this fuzzy logic the best $\mathrm{CH}$ is selected as the $\mathrm{SCH}$, with a reduction in utilization of network overhead and minimal power consumption. This leads to the enhancement in the network lifetime, which in turn increases the reliability and usefulness of the WSN network, where sensors node are provided with minimal battery power and least replacement.

\subsection{Route Selection using Gravitational Search Algorithm}

In our proposed approach, Gravitational Search Algorithm (GSA) is used for selecting an optimum path for transmitting the sensed data to the base station. Using Gravitational Search Algorithm the next best hop transmits the sensed data to the Base Station. Gravitational Search Algorithm is based on Newton's law of gravity, which states that each particle attracts every other particle with a force (say F) which is directly proportional to the product of their mass and inversely proportional to the square of the distance between them".

$$
F=G \cdot\left[\frac{M_{1} M_{2}}{R^{2}}\right]
$$

Where,

$\mathrm{F}$ is the force between the particles, $\mathrm{G}$ is the Gravitational Constant $\left(\mathrm{G}=6.8 \times 10^{-11} \mathrm{~m}^{3} \mathrm{~kg}^{-1} \mathrm{~s}^{-2}\right), \mathrm{M}_{1}$ and $\mathrm{M}_{2}$ are the mass of the two particles respectively, $\mathrm{R}$ is the distance between the particles 
Here each cluster member transmits the sensed data to its respective cluster head and the $\mathrm{CHs}$ send the data to the $\mathrm{SCH}$, which collects the data and compresses it before sending it directly to the base station. Bust in the worst case when a node has minimal remaining battery power and still needs to transmit the sensed data to its $\mathrm{CH}$, during the occurrence of an event of interest, using GSA the best possible path to the $\mathrm{CH}$ is selected .As both the cluster head and cluster members are mobile, due to limited battery power a sensor node might not be in a state to transmit the data (utilizing power) to the connected $\mathrm{CH}$. Hence using GSA it finds the nearest possible node and transmits the data to it, which in turn is transmitted to the respective $\mathrm{CH}$ and then to $\mathrm{SCH}$ to transmit it to the BS. In GSA, the term force is used by the weak sensor (node in WSN) to Choose the next best neighbor (as an intermediate node) to transmit its data to the $\mathrm{CH}$ selected in the given cluster. The force is calculated between the weaksensor node and its nearest neighbor using the formula in Eq. (13).The force of attraction is more for the nearest node and decreases with increase in distance between the nodes. Reliability and transmission efficiency of the selected nearest node increases as the force of attraction between the nodes is at a higher value.

\section{Simulation Result}

The Network Simulator (NS2), is used to simulate the proposed an energy efficient clustering-based routing protocol adopting enhanced cluster formation technique accompanied by fuzzy logic for maximizing the lifetime of a wireless sensor network using 1EEE 802.11 MAC layer. The simulation settings and parameters are summarized in Table 2.

Table 2. Simulation Environment

\begin{tabular}{|l|l|}
\hline No. of Nodes & $10,20,30,40$ and 500 \\
\hline Area Size & $1000 \mathrm{~m}$ X $1000 \mathrm{~m}$ \\
\hline Mac & IEEE 802.11 \\
\hline Simulation Time & $50 \mathrm{sec}$ \\
\hline Traffic Source & CBR \\
\hline Packet Size & 512 \\
\hline Rate & $50 \mathrm{~kb}$ \\
\hline
\end{tabular}

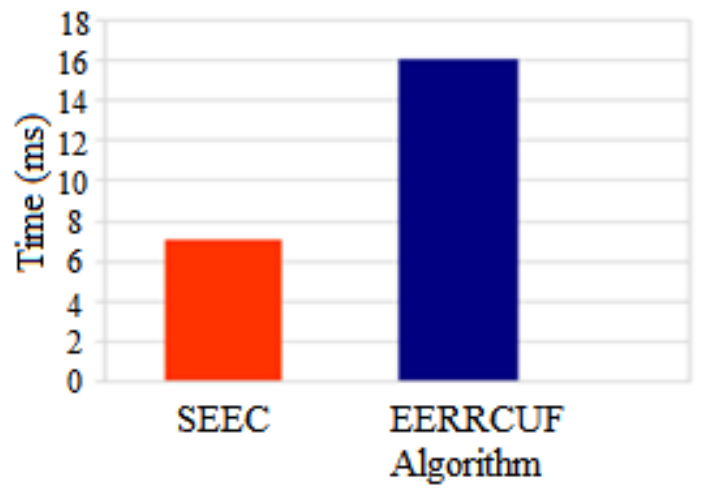

Figure.4 First node dies over time

Since lifespan of a sensor node in a wireless sensor network has a greater influence in determining the overall network lifetime, multiple protocols have been proposed to enhance the lifetime of WSN in an unknown environment where replacement of sensor nodes is an impossible task. Using network simulation it was found that the energy consumption in the proposed EERRCUF is very less compared to the SEEC approach. From fig. 4 the first node is found to die only after 16 seconds in EERRCUF compared to 7 seconds in SEEC. The last node dies in SEEC much before compared to EERRCUF. From fig. 5, it can be observed that the total lifetime of the network employing SEEC, with fixed number of nodes for a fixed amount of time, is found to be 33 seconds. Whereas the lifespan of the overall network employing EERRCUF is found to be 49 seconds. This clearly depicts the stability of the proposed protocol in comparison to the traditional approaches.

The time duration between the death of first sensor node to half nodes of the network depicts the stability and reliability of the given WSN network. More the time duration, the algorithm is found to be more stable than the one with least difference. From fig. 6, it can be observed that half of the nodes in SEEC dies first (by 17 seconds) compared to EERRCUF (36 seconds), where the nodes survive for a much longer time. In SEEC, the time difference between the first nodes death and half nodes death is found to be 10 seconds. Whereas in EERRCUF approach, the time difference is found to be 20 seconds. This clearly depicts the stability of the wireless sensor network utilizing EERRCUF algorithm in comparison to WSN utilizing SEEC protocol. 


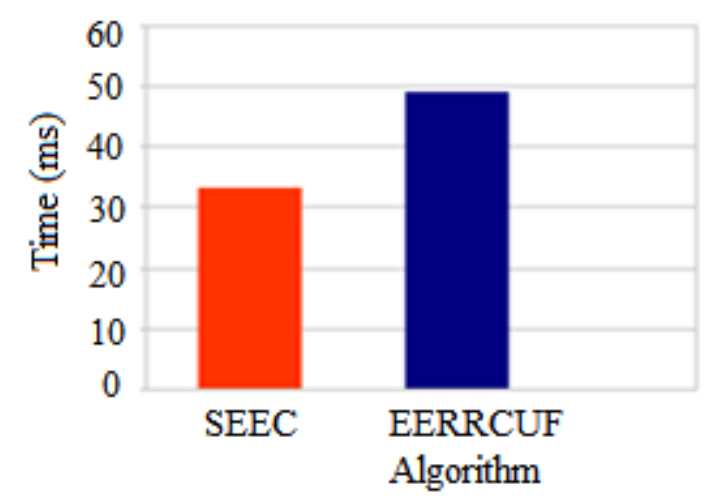

Figure.5 Last node dies over time

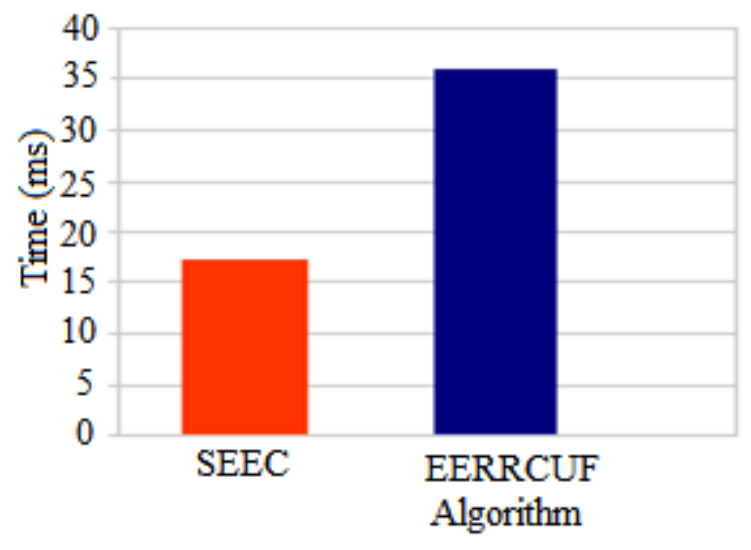

Figure.6 Half node dies over time

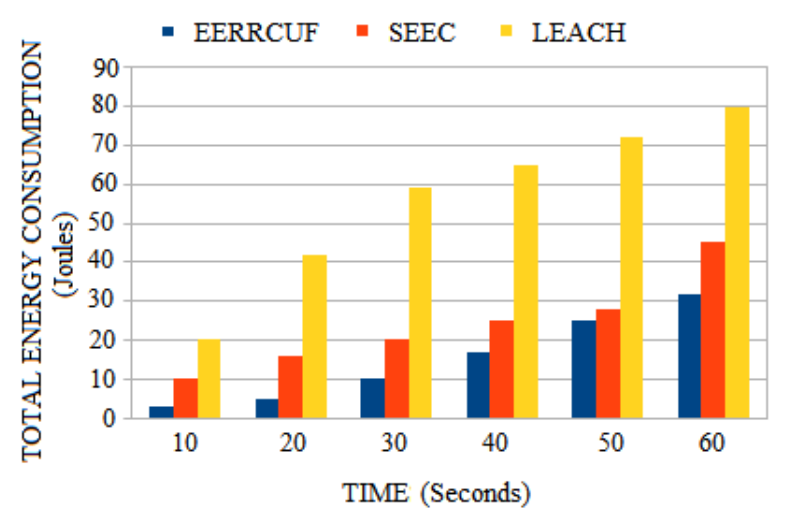

Figure.7 Total Energy Consumption Vs Time

Total energy consumption for the sensor nodes in the given wireless sensor network at different time slot are measured for both the proposed and existing protocol. It is found that with time, there is a gradual increase in the energy consumption for each of EERRCUF, SEEC and LEACH protocol. When the simulation time increases, energy consumption of each node also increases. But from Fig.7, it can be observed that the energy consumption for EERRCUF protocol is very less compared to the existing LEACH and SEEC protocol. Hence, the network lifetime increases with the utilization of EERRCUF protocol in comparison to LEACH and SEEC protocol.

Packet delivery ratio is the ratio between the number of packets received and the number of packets sent. For an efficient network, the delivery ratio should be a maximum value. Higher packet delivery ratio increases the reliability and trustworthiness of the given network. When the simulation time is increased, packet delivery ratio of each node is decreased. But from Fig.8, it can be observed that the packet delivery ratio of the proposed EERRCUF protocol is a far better compared to LEACH and SEEC protocol. From Fig. 9, it is observed that the overall network lifetime of the wireless sensor network can be enhanced by utilizing the EERRCUF protocol in comparison to SEEC and LEACH approach.

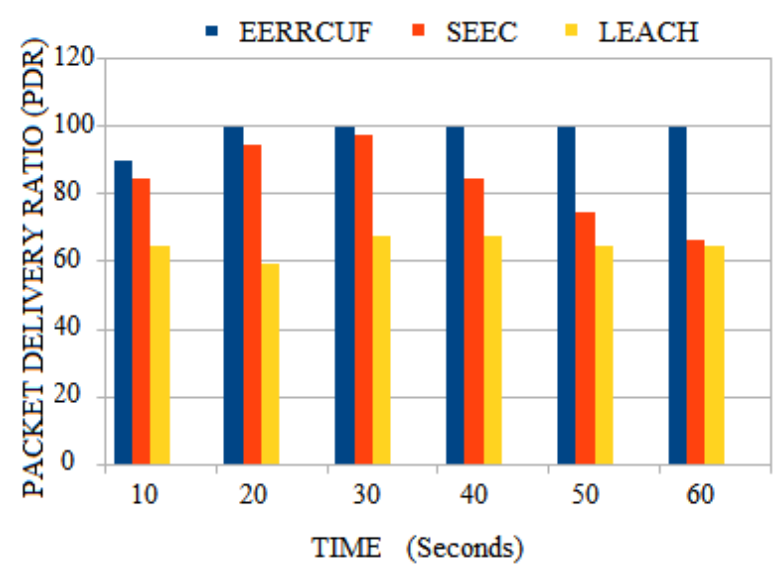

Figure.8 Packet Delivery Ratio Vs Time

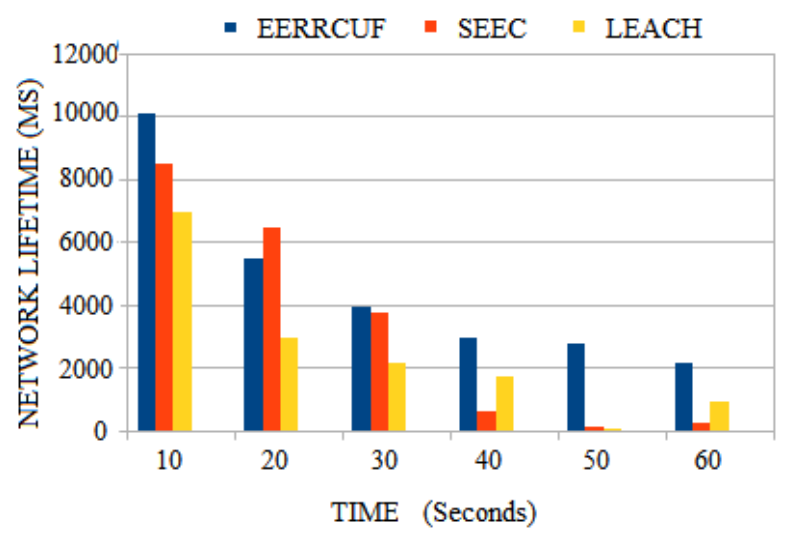

Figure.9 Network lifetime Vs Time 


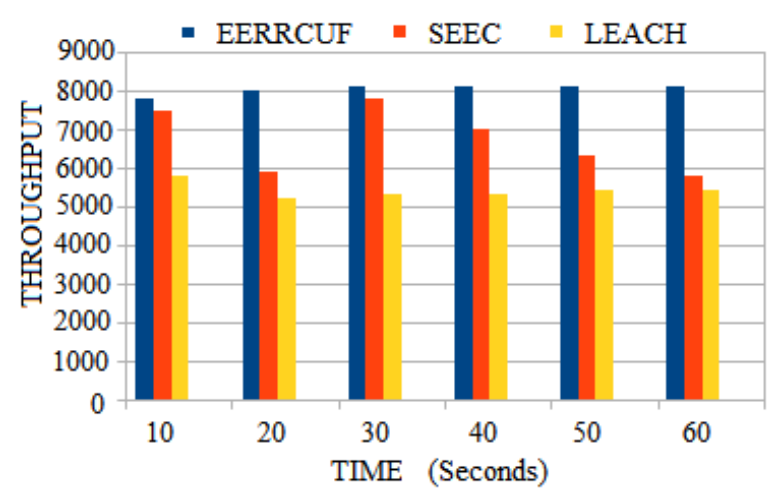

Figure.10 Throughput Vs Time

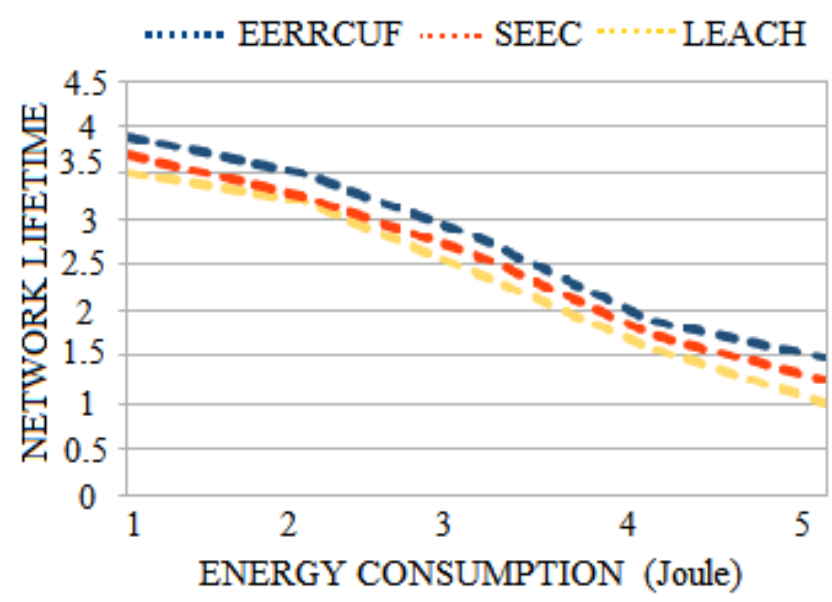

Figure.11 Network Lifetime Vs Energy consumption

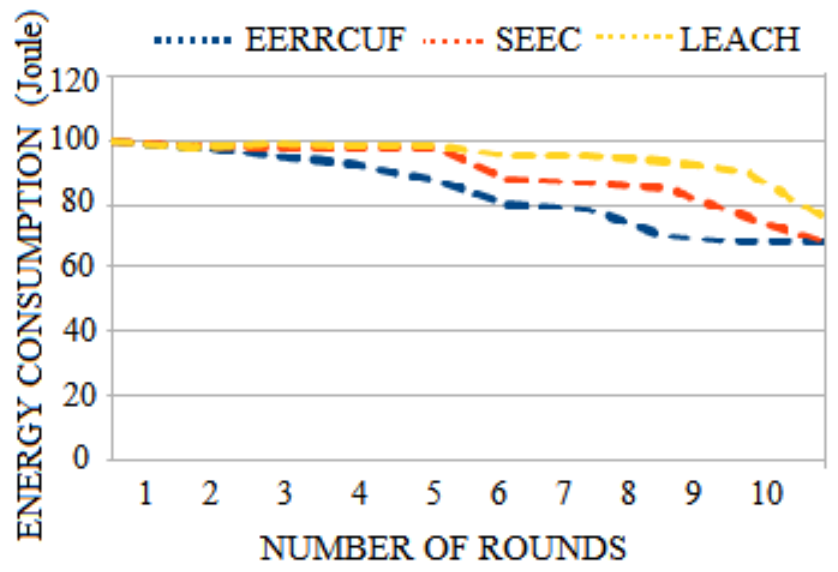

Figure.12 Energy Consumption Vs Number of rounds

Throughput depicts how efficiently a data is being delivered to the destination (base station). It is represented as the number of data being transmitted in bits per second. When the simulation time is increased, throughput the network is decreased. But from Fig.10, the throughput of WSN using EERRCUF protocol is very high compared to the existing approaches. This clearly depicts the reliability and usefulness of the WSN employing EERRCUF protocol.

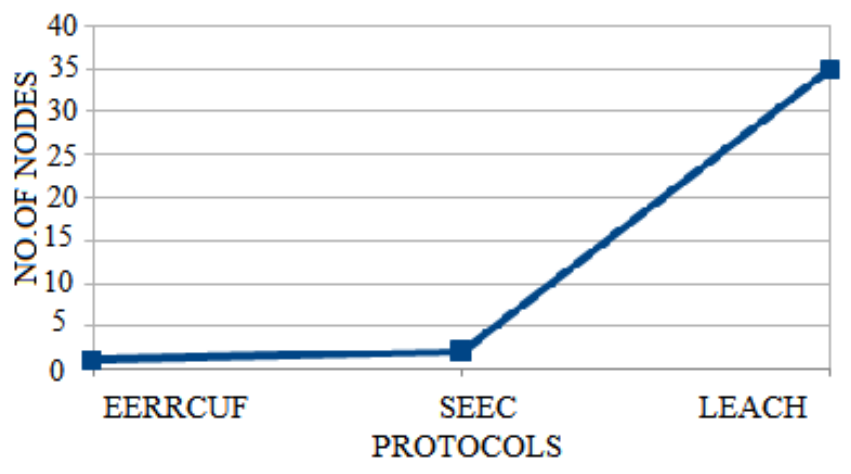

Figure.13 Number of residual nodes

The main goal of an efficient networking protocol is to enhance the lifespan of the given wireless sensor networks (nodes in general) by minimizing the energy consumption at each node. From the network lifetime versus energy consumption comparison, of different protocols, as shown in Fig.12, it is observed that EERRCUF is very stable and enhances the lifespan of the network even at varying number of rounds. Similarly Fig. 11 depicts the network lifetime over varying time slots. EERRCUF is found to enhance the lifespan and provide less latency compared to traditional approaches available in WSN.

Fig. 13 represents the number of residual nodes left in the network without being a cluster member during the clustering process. EERRCUF experiences less number of residual nodes in comparison to SEEC and LEACH approach because of the inclusion of PSO approach in EERRCUF protocol.

\section{Conclusion}

While fuzzy descriptor based LEACH approach seems to be a promising area of development, in this paper efforts have been taken to further enhance the effectiveness of WSN. In our proposed approach an efficient clustering is performed using PSO, where all the nodes are clustered without any residual nodes left in the system. Using the appropriate selection of $\mathrm{CH}$ and SCH the overall networks overhead utilization is reduced and hence the lifetime of the network is enhanced. The result obtained through NS-2 simulation shows that the proposed algorithm 
performs better than SEEC and LEACH approach in terms of stability, reduced energy consumption at each node, less delay and drop at the BS and enhanced the lifetime of the network. As future work, securable routing algorithm will be proposed for efficient routing in the network.

\section{Reference}

[1] T. Singh, J. Singh and S. Sharma, "Energy Efficient Secured Routing Protocol For MANETS", Wireless Netw., pp. 1-9, 2016.

[2] S. K. Singh, M. P. Singh and D. K. Singh, "A Survey of Energy-Efficient Hierarchical Cluster-Based Routing in wireless Sensor Network", Int J. of Advanced Networking and Applications, Vol. 2, No. 2, pp. 570580, August 2010.

[3] R. Raman than and R. Hani, "Topology Control of Multichip Wireless Networks Using Transmit Power Adjustment", Proceeding Nifco, Vol. 2, pp. 404-413, 2000.

[4] F. Farouk, F. Saki and R. Risk, "Multi-level stable and energy-efficient clustering protocol in heterogeneous wireless sensor networks", IET Wireless Sensor Systems, Vol. 4, No. 4, pp. 159-169, 2014.

[5] S. Chand, S. Singh and B. Kumar, "Heterogeneous HEED Protocol for Wireless Sensor Networks", Wireless Pers. Common, Vol. 77, No. 3, pp. 2117-2139, 2014.

[6] B. Zing and Y. Dong, "An improved harmony search based energy-efficient routing algorithm for wireless sensor networks", Applied Soft Computing, Vol. 41, pp. 135-147, 2016.

[7] R. Min, M. Hardwar, S. H. Cho, E. Shih, A. Sinhala, A. Wang and A. Chandrakasan, "Low Power Wireless
Sensor Networks", International Conference on VLSI Design, Bangalore, India, pp. 205-210, January 2001

[8] I. F. Akyildiz, W. Su, Y. Sankarasubramaniam and E. Cayirci, "Wireless sensor networks: a survey", Computer Networks, Vol. 38, No. 4, pp. 393-422, 2002.

[9] N. Javaid, M. Rasheed, M. Imran, M. Guizani, Z. Khan, T. Alghamdi and M. Ilahi, "An energy-efficient distributed clustering algorithm for heterogeneous WSNs", J Wireless Com Network, No. 1, pp.1-11, 2015.

[10]J. R. Parvin and C. Vasanthanayaki, "Particle Swarm Optimization-Based Clustering by Preventing Residual Nodes in Wireless Sensor Networks", IEEE Sensors J., Vol. 15, No. 8, pp. 4264-4274, 2015.

[11]Z. Zahedi, R. Akbari, M. Shokouhifar, F. Safaei and A. Jalali, "Swarm intelligence based fuzzy routing protocol for clustered wireless sensor networks", Expert Systems with Applications, Vol. 55, pp. 313-328, 2016.

[12]P. Nayak and A. Devulapalli, "A Fuzzy Logic-Based Clustering Algorithm for WSN to Extend the Network Lifetime", IEEE Sensors J., Vol. 16, No. 1, pp. 137-144, 2016.

[13]M. Faheem, M. Abbas, G. Tuna and V. Gungor, "EDHRP: Energy efficient event driven hybrid routing protocol for densely deployed wireless sensor networks", Journal of Network and Computer Applications, Vol. 58, pp. 309-326, 2015.

[14]S. K. Dhurandher, M. S. Obaidat, K. Verma, P. Gupta and P. Dhurandher, "FACES: Friend-Based Ad Hoc Routing Using Challenges to Establish Security in MANETs Systems", IEEE Systems Journal, Vol. 5, No. 2, pp. 176-188, June 2011.

[15]J. Branch, B. Szymanski, C. Giannella, R. Wolff and H. Kargupta, "In-network outlier detection in wireless sensor networks", In Proceedings of the 26th IEEE International Conference on Distributed Computing Systems (ICDCS2006), Lisbon, Portugal, 2006. 